\title{
Traducciones vascas del Quijote y de otras obras cervantinas
}

\author{
PATRICIO URQUizU
}

(UNED)

Lo recuerdo paseando por la Concha donostiarra en los años setenta y ochenta. Impoluta la negra sotana. Una camisa almidonada blanca le sobresalía en el cuello. Gafas negras y boina. Lo conocía por la prensa y siempre me había dicho, hor zihoak Kixoteren itzultzailea, ahí va el traductor del Quijote. No le falta mérito. Paseaba ufano, de cháchara con algún otro cura, y de vez en cuando con alguna señora elegante. Recuerdo - si mal no lo recuerdo-, que lo saludé incluso alguna vez. No sé si iba en aquella ocasión con el traductor Ramón Etxezarreta. Era una imagen entre modernista y antiguo régimen.

Pero antes de llegar a Pedro Berrondo y su traducción del Quijote, demos un paseo en compañía de los intentos, fracasos y logros varios de la traducción de la obra cervantina.

1. En 1882 se publicó un breve opúsculo de cuatro páginas sin indicación de lugar, aunque podría ser tal vez el País vasco-francés (Bayona o Biarriz) que se titulaba así: Don Quichotte Manchaco aitoren-seme izpiritutsua Miguel de Cervantes Saavedra deitzen denaz. XLII capitulua (Bigarren partea).

Teniendo en cuenta la forma de Quichotte, es muy posible que el traductor fuera un vasco-francés que tradujera el capítulo XLII de la segunda parte no del original castellano sino de una traducción francesa. Tanto Gotzon Egia ${ }^{1}$ como Jon Benito $^{2}$ la dan por perdida.

2. El año de 1904 se publica en el semanario Pays Basque de Biarriz los días $4,11,18,25$ de junio y 2 de julio de 1904 un ensayo de traducción hecho a petición de un coronel español que, al parecer, debió de ser, según Julián Apraiz, ${ }^{3}$ Don Francisco López Fabra, en su afán de ir completando su obra políglota. Este texto fue reeditado el mismo año, anotado por el vascólogo Edward Spencer Dodgson, con esta portada:

1 Gotzon Egla: On Pedro Berrondo, apez itzultzailea. Internet: www.eizie.org/Argitalpenak/Senez...

2 JON BENITO: Kixotearen euskarazko historia laburra, Berria, 2005-III-8.

"JULIAN APRAIz: Modesto tributo euskaro rendido a Cervantes en el tercer centenario de la aparición del Ingenioso Hidalgo D. Quijote de la Mancha, Vitoria, Domingo Sar, 1905. 
MIGUEL SAAVEDRA CERVANTESTARRAK/scribatu zuen/DON KIXOTEN GERTHAKARIAK/deitzen den liburuko/aintzin-solas eta hari darreitzan hirur buruchoak/DUVOISIN capitainak/Zubiburun zegoela Laphurdiko Heuskaraz emanak./Erneste SEITZ imprimazale, BIARRITZEN/1904-garren Hurteko uztailan. [Prólogo y tres capítulos primeros de Las aventuras de Don Quijote escritas por Miguel de Cervantes Saavedra , traducidos por el capitán Duvoisin al euskera de Laphurdi, cuando estaba en Zubiburu (Ciboure). Impresor Ernest Seitz, Biarriz, julio de 1904].

Si tenemos en cuenta que Jean Duvoisin (Ainhoa 1810-1891), fue el autor de la primera traducción completa de la Biblia al dialecto labortano bajo el título de Bible edo Testament zahar eta berria.... (Londres, 1859-1865), edición hecha a expensas del vascólogo Louis Lucien Bonaparte, sobrino del emperador Napoleón III, no nos queda sino lamentar que el coronel español no fuera tan espléndido como el lingüista francés.

Tradujo Duvoisin también otras obras, como el Kempis, que dejó inédito e incompleto y fue editado por Haristoy (Jesu Kristoren imitazionea, Pau 1896), y el Telémaco de Fenelón que edité ${ }^{4}$ hace algunos años.

Decimos que es de lamentar no siguiera con su traducción del Quijote pues su prosa ha sido considerada como un bello modelo, tanto en las traducciones que llevó a cabo, como en las que dejo inconclusas o en la creación de Laborantzako liburua edo bi aita semeren solasak laborantzaren gainean [El libro de la agricultura o Diálogos entre padre e hijo sobre la agricultura] (Bayona, 1858).

Es evidente que Duvoisin no pudo revisar su traducción antes de la edición de su obra y que las notas y correcciones hechas por el vascólogo Dodgson a veces son pertinentes y otras no, empezando por la primera correción que dice haber hecho: (1) Le manuscrit porte den bezala au lieu de zeren et baita... Bezala signifie comparaison, et non temps ni cause.

"Zeren ene burutik atheratua baita", dice la edición corregida del manuscrito que fue entregado por Arturo Campión a Dodgson y por éste a la Biblioteca Nacional de París (MS Basques et Celtes n. ${ }^{\circ} 130$ ), a cambio de 5 francos. Es evidente para un nativo de la lengua vasca, que la forma primitiva no necesitaba ninguna corrección. Menos mal que las notas de Dodgson nos muestran como era el original.

Este opúsculo editado en Biarriz en 1904 fue reeditado por Apraiz en Vitoria en 1905. En esta nueva edición Dodgson pedía al nuevo editor que le daba permiso para la nueva edición pero que respetara las notas. El vitoriano editó el texto con las correcciones llevada a cabo por el vascólogo inglés pero sin las notas.

3. En la edición de Apraiz, sin embargo, además de la traducción de Duvoisin se dan otras versiones como son:

${ }^{4}$ PATRI URkizu (ed.): Fenelon-Duvoisin, Telemake: Ulisen semearen gertaerak, Elkar, Donostia 1988. 
- Segunda parte del Quijote, principio del capítulo XLII por don Pablo Zamarripa en guipuzcoano y vizcaino mezclados (47-52).

- Conclusión del cap. XLII ó primeros consejos de D. Quijote á Sancho por el M. I. Sr. D. Mateo Múgica (52-57).

- Principio del cap. XLII, en dialecto vizcaino, por D. Evaristo Bustinza (57-59).

- Fín del cap. XLIII, en dialecto alavés, por D. Serafín Ascasubi (59-64)

- Principio del cap. XLII en labortano (65-71).

- Cincuenta refranes del Quijote en vascuence y castellano por D. Pablo Zamarripa (71-78).

Parece bastante claro que estas aportaciones tienen más valor lingüístico (dialectológico sobre todo) y paremiológico que extrictamente literario.

4. En 1928 la sociedad Euskalzaleak organiza un concurso en el que se ha de traducir el capítulo IX del Quijote, que habla de la estupenda batalla que tuvieron el gallardo vizcaino y el valiente manchego. Se presentaron diversos traductores (Agustín Anabitarte, Fray Buenaventura Oyeregui, Jon Garbizu, Xabier Agirre «Lizardi», Angel Irigaray, Nicolás Ormaetxea "Orixe») y entre ellos resultó ganador Nicolas Ormaechea «Orixe», cuya traducción así como las de Lizardi, Irigaray y Anabitarte se publicaron en la Revista Internacional de Estudios Vascos (RIEV) en 1928 y 1929. He aquí cómo titularon el capítulo:

- Ormaechea, N.: «Orixe», Euskaldun bipilak eta Manchatar bizkorrak izan zuten burrukaldi lazgarriaren ondarra. (RIEV, 1929, 6-9).

- AgrRre, X.: «Lizardi», Euskaldun zutiñak eta mantxar bulardetsuak alkarrekin egindako burruka arrigarria amaitu ta azkentzea. (RIEV, 1929, 207-211).

- ANGEl IRIGARAY: Non etsia ematen zaion euskaldun lerdenak eta mancha'tar kementsuak elkarrekin izan zuten arrigarrizko garaikeriari. (RIEV, 1928, 598-607).

- Agustín Anabitarte: Euskaldun lerdenak eta Mantxa'ko seme bioztunak izandako arrigarrizko borroka bukatu ta azkena ematen zaionekoa. (RIEV, 1928, 608-612).

Dice Irigaray en una nota a la traducción que algunas oraciones que le parecen un tanto largas las ha cortado pues de ese modo no se pierde la materia de la narración, ya que como señala Jacinto Benavente para traducir bien no hay que hacerlo literalmente sino que lo que quiere decir el autor trasladarlo lo más clara y hermosamente posible.

Así también señala Lizardi lo siguiente a propósito de la traducción:

Itzulpen ontan alegiña egin da itzulgaiari aalik eta bertenetik jarraitzen; baña noizik-bein, zeatzegi itzuliak esaldia gogortu zezakenean, zertxobait, beraizik, aldatu izan da. Bestalde, gaztelerak ain berezko dituan esaldi luze alkar-lotuerantsiak nolabait moztu ta banatzera ekin diogu, euskerari obato dagokion la- 
burtasuna aal-neurrian jaretsi-eske. Azkenik, itz gaitz ala berri batzuen ondoan zaar-errezagoak ere adirazi dira, batera naiz besterako aukera egon dedin. [Se ha intentado en esta traducción seguir lo más de cerca al original, pero de vez en cuando al traducir demasiado literalmente quedaba la frase muy dura y por lo tanto se ha cambiado. Por otro lado, las frases largas y llenas de subordinadas que son tan propias del castellano las hemos cortado, pues el laconismo le es más propio al euskera, a la búsqueda de más libertad. En fin al lado de palabras difíciles o nuevas hemos indicado otras añejas más fáciles, a fin de que pueda elegirse la que plazca.]

Es evidente que el poeta Xabier Agirre «Lizardi» (1896-1933) estaba muy preocupado porque saliera una traducción acorde con el espíritu del euskera, más que con la literalidad del texto. Y que Nicolás Ormaechea «Orixe» (18881961), autor de la gran obra Euskaldunak [Los Vascos, 1950], exjesuita, hombre de sólida formación clásica, traductor de Las Confesiones de San Agustín, de poemas de San Juan de la Cruz, del Lazarillo de Tormes, del Mireio de Federico Mistral, consiguió dar al euskera en palabras de Luis Michelena un nuevo tono y una nueva musicalidad.

Veamos comparativamente cómo traducen los sintagmas de Cervantes $G a$ llardo vizcaino/valiente manchego:

Orixe: euskaldun bipilalmanchatar bizkorra.

Lizardi: euskaldun zutinalmantxar bulardetsua.

Anabitarte: euskaldun lerdenalMantxa'ko seme bioztuna.

Irigaray: euskaldun lerdenalmancha'tar kementsua.

Como se puede observar hay unanimidad en la traducción de vizcaino como euskaldun, mientras que las variantes son claras en los epítetos que sustituten a gallardo y valiente.

5. Hacia 1930 se hicieron diversas traducciones de las Novelas ejemplares de Cervantes, que no se editaron y se hallan en el Fondo Urquijo de la Biblioteca Koldo Mitxelena de Donostia, y que son las siguientes:

Lucio Berasaluze Mendaro, Rinconete y Cortadillo (091 Mss 49)

Pablo Fermin Yrigaray, Andere Kornelia (091 Ms 26)

Basauri, Andre Korneli (091 AIT doc 85)

XX, Korneli Andrea (091 Ms 27)

La Señora Cornelia había sido editada con un estudio preliminar por Apraiz, en el que estudiaba a fondo las dos figuras escolares que aparecen en ella: Isunza, en recuerdo del Proveedor vitoriano Pedro Martinez de Isunza y Lequeitio, en quien Cervantes encontró en Andalucía más que un jefe un amigo, y Juan Gamboa, que decidieron dejar los estudios de Salamanca e irse a Flandes...

Del médico -cirujano del Hospital de Pamplona-, y escritor Irigarai, que escribió unas interesantes memorias sobre la guerra civil tituladas Gerla urte 
gezur urte 'Año de guerras, año de mentiras', 1994), y que también tradujo el capítulo IX existe un fragmento de La Gitanilla traducida como Ijitotxua. ${ }^{5}$

Kindin Muxika en su exilio de Buenos Aires el año de 1948 tradujo en un bello euskera el capítulo LXXIV, es decir el final de la obra, que no se publicó hasta 1978 en Boletín del Instituto Americano de Estudios Vascos. ${ }^{6}$

Unos años antes Gabino Garriga (Bilbao, 1885 - La Plata, 1969). También editó en dialecto vizcaino y con gran influencia sabiniana diversos capítulos del Quijote y precisamente los de la Insula Barataria. Dice así:

Kiyote Yaunaren 2-garren zatiko XLV, XLVII, XLIX, LI eta LII gn. Atalak edo: Nola Barataria ugarteko jaurbide Panza' tar Santxo' ren eskuetan erabilli zan... XLV gn. Atala. Nola Panza'tar Santxo'k bere ugarteko yabetza artu zun eta yaurtzen asi zan. ${ }^{7}$

6. Y por fin llegó Pedro Berrondo, que tradujo el Quijote completo, editando el primer tomo en 1976 en la editorial Itxaropena con la ayuda de la Caja de Ahorros de Donostia, y la obra completa en 1985 con la subvencion de la Diputación Foral de Gipuzkoa. ${ }^{8}$

Cuando le preguntaron en una entrevista a Berrondo porqué llevó tamaña empresa a cabo dedicándole tantas horas de su vida — dos o tres horas por página, aproximadamente tres mil horas - la contestación fue:

Maitasunak barkatzen ez duelako, es decir, porque el amor no perdona ${ }^{9}$... no escribo en euskera porque esta lengua tenga una larga o corta vida, sino porque es mi vida... No me duele el haberle dedicado tantas horas al Quijote, al contrario estoy muy orgulloso de ello.. Al traducir lo más fácil son los diálogos. Luego también algunas descripciones, ahi, sin embargo ya hay un límite cuando se trabaja con conceptos un tanto abstractos la cosa se pone más dificil. Un trabajo de este tipo exige introducirse en lo más íntimo del euskera y aprender muchas cosas. Pero es más fácil traducir un libro como el Quijote que cualquier autor modernol...IEl País Vasco debiera hacer examen de conciencia y las paces con Castilla. Los vascos tienen cierta tendencia hacia Castilla, hacia Salamanca. Ahora, sin embargo se va en búsqueda de luces a Alemania....

7. Lucas Dorronsoro es otro traductor que además de traducir los cuentos fantásticos de Ruben Darío, y las obras teatrales de Jardiel Poncela (Udaberri gau artan 'Aquella noche de primavera'), Joaquín Calvo (Harresi latza 'La Muralla') de Alejandro Casona (Zazpi deadar itsasoan 'Siete gritos en el mar), etc., ha tra-

5 A. IRIGARAY: «Una versión euskérica de Cervantes», Fontes Linguae Vasconum, 1980, 471-473.

${ }^{6}$ KINDIN MuXIKA: «Miguel de Cervantes Saavedra'ren Don Quijote. LXXIV. Don Quijote nola gaxotu zan, bere azkengogoa, ta nola il zan», Boletín del Instituto Americano de Estudios Vascos, (BIAEV), 1978, vol. 29, 139-141, 189-190.

7 BIAEV: 1963, 123-125; 166-168; 1964, 35-37, 88-91, 126-127.

${ }^{8}$ Cervantes SaAvedra, Miguel (1976-1985): Don Kijote Mantica' ko, Donostia, José Estornes Lasa.

${ }^{9}$ «Solasean. Berrondo», Oh! Euzkadi, 2, Donostia, 1980-Ekaina. 
ducido y publicado en la La primitiva Casa Baroja de San Sebastián entre Las Novelas Ejemplares, las siguientes: Rinkonete eta Kortadillo. Kristal lizentziatua (1987); Ijito neska [La gitanilla] (1988); Txukundari argia (1989); Gezurrezko ezkontza eta Zipione eta Berganzaren elkarrizketa (1990).

8. No olvidemos que también Gabriel Aresti (Bilbao, 1933-1975), poeta, ensayista y autor dramático, fue un gran traductor (Chaucer, Boccaccio, Baudelaire, Castelao, Lorca, Hikmet...) y que tradujo los primeros capítulos del Quijote (Susa, Itzulpenak, 1986).

9. Este cuarto centenario de la primera edición de El Quijote están previstos en el País Vasco algunos actos en honor de Cervantes, como un curso de verano, un número exclusivo de la revista literaria Egan, la reedición de la traducción de Berrondo por la Real Sociedad Vascongada de los Amigos del País en una edición más barata aunque se mantendrán las ilustraciones de los veintiocho dibujantes y pintores que participaron en la obra entre los que se hallan Arbeloa, Momoitio, Apezetxea, Gonzalo Txillida, entre otros.

10. De todos modos sería de agradecer que el nuevo editor con criterios filológicos científicos hiciera las correcciones pertinentes en textos como el verso del Canto XXXV del Orlando Furioso de Ariosto con el que acaba el Primer Tomo, ya que aparece como Forse altri kantera kon miglior plettro (sic!), en lugar de Forsi altro canterà con miglior plettro. Y que los consejos políticos de Berrondo se tuvieran más en cuenta. 